\title{
Constraining Connectivity to Encourage Modularity in HyperNEAT
}

UCF Dept. of EECS Technical Report CS-TR-10-10

\author{
Phillip Verbancsics and Kenneth O. Stanley \\ Department of Electrical Engineering and Computer Science \\ University of Central Florida \\ Orlando, FL 32816, USA \\ \{verb,kstanley\}@eecs.ucf.edu
}

November 2010

\begin{abstract}
A challenging goal of generative and developmental systems (GDS) is to effectively evolve neural networks as complex and capable as those found in nature. Two key properties of neural structures in nature are regularity and modularity. While HyperNEAT has proven capable of generating neural network connectivity patterns with regularities, its ability to evolve modularity remains in question. This paper investigates how altering the traditional approach to determining whether
\end{abstract}


connections are expressed in HyperNEAT influences modularity. In particular, an extension is introduced called a Link Expression Output (HyperNEAT-LEO) that allows HyperNEAT to evolve the pattern of weights independently from the pattern of connection expression. Because HyperNEAT evolves such patterns as functions of geometry, important general topographic principles for organizing connectivity can be seeded into the initial population. For example, a key topographic concept in nature that encourages modularity is locality, that is, components of a module are located near each other. As experiments in this paper show, by seeding HyperNEAT with a bias towards local connectivity implemented through the LEO, modular structures arise naturally. Thus this paper provides an important clue to how an indirect encoding of network structure can be encouraged to evolve modularity.

\section{Introduction}

As generative and developmental systems (GDS) are asked to create increas-

ingly large and complex structures, the question of how to organize and constrain such structures becomes increasingly important. Neural networks in nature exhibit several important organizational principles, such as modularity, regularity, and hierarchy [10, 12, 21], which enable evolution to scale structures in complexity and size. Such features might accordingly enable evolutionary algorithms to similarly scale to problems of high complexity and dimensionality if they could be encouraged to emerge. This paper shows how modularity can be encouraged as a function of geometry through an indirect 
encoding.

The challenge in encouraging modularity is to bias the search towards modular solutions without restricting the search space to modularity alone. The danger in explicitly designing modularity into the representation is that it may bias the search towards modular structure when non-modular designs may be required, inhibiting search from finding an optimal solution [6]. For example, fully-connected artificial neural networks (ANNs) are completely non-modular. To produce a modular ANN, connectivity would be restricted, but that would prevent solutions that may require full connectivity.

Hypercube-based NeuroEvolution of Augmenting Topologies (HyperNEAT; $[8,9,14])$ has been shown to generate regularities in ANN weight patterns $[8,9,14,20]$, but Clune et al. [1] showed that it struggles to produce modular connectivity patterns. To address this problem, this paper introduces a novel insight that can consistently bias HyperNEAT towards modular structures. The initial idea is that a bias towards locality is an important prerequisite to modularity. However, locality alone is not enough because not all connectivity should be local. Thus the key insight is that the search should start with a bias towards locality that can later be adjusted by evolution to different levels for different parts of the network geometry. This idea is implemented and tested in this paper in a variant of HyperNEAT called HyperNEAT with Link Expression Output (HyperNEAT-LEO).

To demonstrate its advantage, HyperNEAT-LEO is compared to the standard HyperNEAT method and another variant that enforces an external distribution on connectivity. The HyperNEAT variants are investigated in the Retina Left and Right problem [1] (based originally on Kashtan and Alon 
[11], in which the ANN must indicate whether particular objects are detected in the left and right retinas). The Retina problem is modular because the calculations for the left and right sides are independent, which has proven difficult for HyperNEAT to represent [1]. In this task, a significant difference is demonstrated among the variants. The standard HyperNEAT does not perform well at the task, finding a solution only $12 \%$ of the time or less. The imposed connectivity distribution also performs poorly. However, interestingly, HyperNEAT-LEO, which evolves the pattern of expressed connections, is able to achieve up to a $91 \%$ success rate if it begins with a bias towards local connectivity. These results demonstrate that modularity can be encoded as a function of geometry, providing a means to evolving modularity in HyperNEAT.

\section{Background}

The geometry-based methods that underlie the approach to modularity are reviewed first and modularity is then discussed.

\subsection{NeuroEvolution of Augmenting Topologies (NEAT)}

This section briefly reviews the NEAT evolutionary algorithm [15, 17], a popular policy search method that evolves ANNs. It is also the foundation for the HyperNEAT method. NEAT evolves connection weights as well as adds new nodes and connections over generations, thereby increasing solution complexity. It has been proven to be effective in challenging control and decision-making tasks $[17,18,22,23]$. NEAT starts with a population 
of small, simple ANNs that increase their complexity over generations by adding new nodes and connections through mutation. That way, the topology of the network does not need to be known a priori; NEAT searches through increasingly complex networks as it evolves their connection weights to find a suitable level of complexity. The techniques that facilitate evolving a population of diverse and increasingly complex networks are described in detail in Stanley and Miikkulainen [15]; Stanley and Miikkulainen [17]; the important concept for the approach in this paper is that NEAT is a policy search method that discovers the right topology and weights of a network to maximize performance on a task. The next section reviews the extension of NEAT called HyperNEAT that allows it to exploit geometry through representation.

\subsection{CPPNs and HyperNEAT}

NEAT is an appropriate platform to study modularity in GDS because it easily extends to an indirect encoding, which means a compressed description of the solution network. Such compression makes the policy search practical even if the state space is high-dimensional. One effective indirect encoding is to compute the network structure as a function of geometry. This section describes such an extension of NEAT, called Hypercube-based NEAT (HyperNEAT; $[8,9,14]$ ), which enables high-dimensional static representations. The effectiveness of the geometry-based learning in HyperNEAT has been demonstrated in multiple domains and representations, such as checkers

$[8,9]$, multi-agent predator prey [3, 5], visual discrimination [14], quadruped locomotion [2], and RoboCup Keepaway [20]. A full description of Hyper- 
NEAT is in Gauci and Stanley [8, 9] and Stanley et al. [14].

The main idea in HyperNEAT is that geometric relationships are learned though an indirect encoding that describes how the weights of the ANN can be generated as a function of geometry. Unlike a direct representation, wherein every connection in the ANN is described individually, an indirect representation describes a pattern of parameters without explicitly enumerating each such parameter. That is, information is reused in such an encoding, which is a major focus in the field of GDS from which HyperNEAT originates $[16,19]$. Such information reuse allows indirect encoding to search a compressed space. HyperNEAT discovers the regularities in the geometry and learns a policy based on them.

The indirect encoding in HyperNEAT is called a compositional pattern producing network (CPPN; [13]), which encodes the weight pattern of an ANN $[8,14]$. The idea behind CPPNs is that geometric patterns can be encoded by a composition of functions that are chosen to represent common regularities. Given a function $f$ and a function $g$, a composition is defined as $f \circ g(x)=f(g(x)))$. In this way, a set of simple functions can be composed into more elaborate functions through hierarchical composition (e.g. $f \circ g(f(x)+g(x)))$. For example, the Gaussian function is symmetric, so when it is composed with any other function, the result is a symmetric pattern. The internal structure of a CPPN is a weighted network, similar to an ANN, that denotes which functions are composed and in what order. The appeal of this encoding is that it can represent a pattern of connectivity, with regularities such as symmetry, repetition, and repetition with variation, through a network of simple functions (i.e. the CPPN), which means that instead of 
evolving ANNs, NEAT can evolve CPPNs that generate ANN weight patterns.

Formally, CPPNs are functions of geometry (i.e. locations in space) that output connectivity patterns whose nodes are situated in $n$ dimensions, where $n$ is the number of dimensions in a Cartesian space. Consider a CPPN that takes four inputs labeled $x_{1}, y_{1}, x_{2}$, and $y_{2}$; this point in four-dimensional space can also denote the connection between the two-dimensional points $\left(x_{1}, y_{1}\right)$ and $\left(x_{2}, y_{2}\right)$. The output of the CPPN for that input thereby represents the weight of that connection (figure 1). By querying every pair of points in the space, the $\mathrm{CPPN}$ can produce an ANN, wherein each queried point is the position of a neuron. While CPPNs are themselves networks, the distinction in terminology between CPPN and ANN is important for explicative purposes because in HyperNEAT, CPPNs encode ANNs. Because the connection weights are produced as a function of their endpoints, the final pattern is produced with knowledge of the domain geometry, which is literally depicted geometrically within the constellation of nodes.

In effect, the CPPN paints a pattern within a $n$-dimensional hypercube that is interpreted as an isomorphic connectivity pattern. Weight patterns produced by a CPPN in this way are called substrates so that they can be verbally distinguished from the CPPN itself. It is important to note that the structure of the substrate is independent of the structure of the CPPN. The substrate is an ANN whose nodes are situated in a coordinate system, while the CPPN defines the connectivity among the nodes of the ANN. The experimenter defines both the location and role (i.e. hidden, input, or output) of each node in the substrate. 


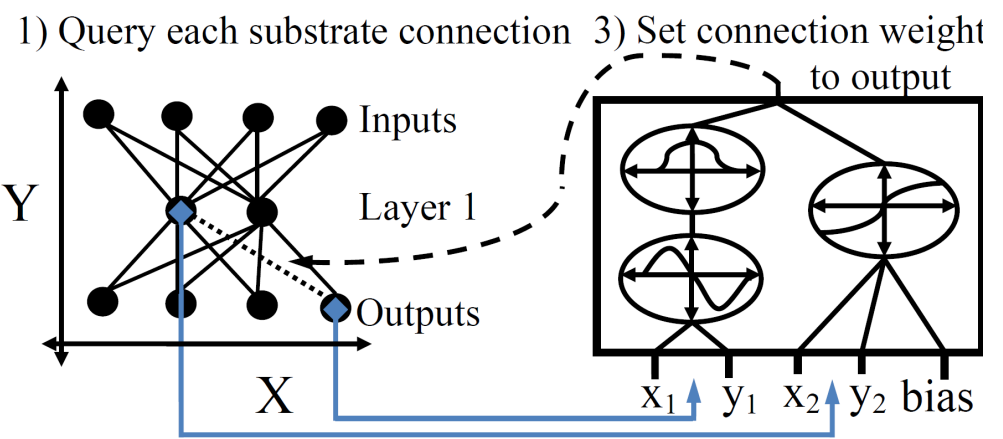

2) Feed coordinate pair to CPPN ANN Substrate

CPPN

Figure 1: A CPPN Describes Connectivity. A grid of nodes, called the ANN substrate, is assigned coordinates. (1) Every connection between layers in the substrate is queried by the $\mathrm{CPPN}$ to determine its weight; the line connecting layers in the substrate represents a sample such connection. (2) For each such query, the CPPN inputs the coordinates of the two endpoints, which are highlighted on the input and output layers of the substrate. (3) The weight between them is output by the CPPN. Thus, CPPNs can generate regular patterns of connections.

As a rule of thumb, nodes are placed on the substrate to reflect the geometry of the domain (i.e. the state), making setup straightforward [2, 9, 14]. For example, a visual field can be laid out in two dimensions such that nodes that receive input from adjacent locations in the image are literally adjacent in the network geometry. This way, the pattern of weights becomes a direct function of the domain geometry, which means that knowledge about the problem can be injected into the search and HyperNEAT can exploit the 
regularities (e.g. adjacency, or symmetry, which the CPPN sees) of a problem that are invisible to traditional encodings. For example, geometric knowledge can be imparted e.g. by including a hidden node in the CPPN that computes $\operatorname{Gauss}\left(x_{2}-x_{1}\right)$, which encodes the concept of locality and symmetry on the $x$-axis, an idea employed in the implementation in this paper. This idea can be illustrated by considering three source points (i.e. nodes) with $x$ positions $-1,0,1$ and one target point at $x=0$.

$$
\begin{gathered}
w_{1}=\operatorname{Gauss}(0+1)=0.37 \\
w_{2}=\operatorname{Gauss}(0-0)=1 \\
w_{3}=\operatorname{Gauss}(0-1)=0.37
\end{gathered}
$$

Thus, the output of this function is both symmetric about the $x$-axis and decreasing with distance from the target.

In summary, HyperNEAT evolves the internal topology and weights of the CPPN that compactly encodes ANN weight patterns. The process is shown in Algorithm 1. Next, modularity is discussed.

\section{$2.3 \quad$ Modularity}

Modularity can be defined as the separability of structure into functionally

independent units $[1,11,12]$. Modular designs are common to both biological and engineering systems. For example, cells assemble into tissues, tissues into organs, and organs into organisms. Decomposing a design into modules can allow evolutionary algorithms to address complex tasks that require subfunctions of the solution to be independently optimized [11]. Approaches to modularity in evolutionary algorithms range from explicitly encapsulating 
Input: Substrate Configuration

Output: Solution CPPN

Initialize population of minimal CPPNs with random weights;

while Stopping criteria is not met do

foreach $C P P N$ in the population do

foreach Possible connection in the substrate do

Query the CPPN for weight $w$ of connection;

if $A b s(w)>$ Threshold then

Create connection with a weight scaled proportionally to $w$ (figure 1 );

end

end

Run the substrate as an ANN in the task domain to ascertain fitness;

end

Reproduce CPPNs according to the NEAT method to produce the next generation;

end

Output the Champion CPPN.

Algorithm 1: Basic HyperNEAT Algorithm

modules $[6,24]$ to alternating tasks to facilitate their discovery [11].

A common approach to modularity in evolutionary algorithms is to preserve or create modules through high-level processes. For example, modularitypreserving representations preserve the modularity that exists in the phenotype space by transferring it to the genotype space through encapsulation $[6,24]$. The process of encapsulation creates new genotypic primitives that are groupings of existing primitives. In this way, modules can be generated 
and assembled together to produce solutions. Such high-level processes for modularity are dependent on the capability of the algorithm to select beneficial modules and may be detrimental to search by creating modules that are not useful.

Alternatively, the evolutionary algorithm may be modified such that fitness pressure is applied to encourage discovering modularity. In modularly varying goals evolution [11], the task that is being solved alternates at set intervals during evolution. Bias towards modularity is created by selecting tasks in which there are shared sub-goals and it is easier to switch between tasks if the appropriate modules for these sub-goals are discovered. For example, the modularly varying goals version of the Retina Problem [11] switches between the tasks of (1) determining if there is a target pattern in the right or left retina and (2) determining if there is a target pattern in the right and left retina. Both of these tasks must independently discover the appropriate functions for the left and right retinas, and then combine them correctly depending on the task. The challenge is to select tasks that share modules such that their discovery is beneficial.

Clune et al. [1] applied HyperNEAT to the modularly-varying goals Retina Problem. Standard HyperNEAT struggled to produce modularity in this task and achieved significantly lower correct classifications than the direct encoding implemented by Kashtan and Alon [11]. In addition to the modularlyvarying goals version of the Retina Problem, HyperNEAT was also tested in the non-varying Retina Problems, i.e. the fixed goal of right and left and the fixed goal of right or left, and alternative Retina Problems that must judge each retina's input individually. For example, a left output indicates validity 
for the left pattern. The most difficult of these tasks for HyperNEAT is the Retina Left and Right task [1], which must correctly judge as valid or invalid each retina's pattern separately. Interestingly, Clune et al. [1] found that imposing modularity by disabling connections between the left and right sides of the network improved HyperNEAT's performance [1]. This enforced modularity demonstrates that modularity could potentially provide HyperNEAT an advantage in this task, but that HyperNEAT struggles in the creation of modularity on its own.

This paper adds to our understanding of modularity by focusing on the role of geometry in encoding modularity. The next section explains modifications to HyperNEAT based on this idea that alter how connection expression is controlled.

\section{Thresholding in HyperNEAT}

Although the HyperNEAT method succeeds in a number of challenging tasks $[8,9,14,20]$ by exploiting geometric regularities in the solution, it is less effective at generating modular solutions [1]. Instead it tends to generate fully-connected networks even when more limited connectivity would be beneficial. The conventional method for controlling connectivity in HyperNEAT is a threshold that limits the range of values output by the CPPN that can be expressed as weights $[9,14]$. The threshold is a parameter specified at initialization that is uniformly applied to all connections queried. When the magnitude of the output of the CPPN is below this threshold, the connection is not expressed. In this way, the amount of connections expressed may be 
controlled by raising or lowering the threshold when fewer or more connections are desired. Because the threshold is uniform across all connections, it influences every connection equally with no bias towards modularity (which likely requires different levels of connectivity in different areas).

As an alternative to the traditional uniform threshold, one idea to control connection expression is a dynamic threshold. The dynamic threshold varies depending on the specific nodes or the connection being queried. As each connection is queried, the threshold, which determines whether the CPPNgenerated weight should be expressed as a connection, is updated and then applied. In this way, a particular expression distribution that is determined a priori can be enforced to influence the pattern of connectivity in the ANN. In particular, this paper explores a dynamic threshold based on distance with the value $t=c+c *\left\|\mathbf{n}_{i}-\mathbf{n}_{j}\right\|$, where $t$ is the local threshold value, $c$ is a constant parameter set at initialization and $\left\|\mathbf{n}_{i}-\mathbf{n}_{j}\right\|$ is the Euclidean norm of the difference between the endpoint-nodes' coordinate-vectors. This threshold makes sense for modularity because it expresses the concept of $l o-$ cality by discouraging connections of greater distance. That is, the higher the distance is between nodes, the higher the threshold will be.

In another alternative to the uniform threshold, instead of thresholding connections through an external value, HyperNEAT itself is extended to generate an expression pattern that controls whether connections are expressed at different locations independently of their weights. For this purpose, a new Link Expression Output (LEO) is added to the CPPN that indicates whether a connection is expressed. When the LEO (which is a step function) is greater than 0.0 the connection weight is set to the usual CPPN weight 
output; otherwise it is not expressed (Algorithm 2). In this way, the pattern of connectivity can be controlled by the CPPN itself independently of the weights, and that connectivity can be evolved as a function of geometry. This approach follows the idea that connection expression and weights are best considered separately.

Furthermore, because expression patterns are functions of geometry (as are the weights), evolution can be seeded with geometric principles that bias the pattern of connectivity. Evolution is seeded by initializing CPPNs in the first generation with seed topologies that represent important concepts. For example, locality can be expressed through a Gaussian function. Because the Gaussian function peaks when its input is 0.0 , inputting a difference between coordinates, e.g. $\Delta x$, achieves the highest value when the coordinates are the same. To seed for locality on multiple dimensions, a Gaussian node can be added to the seed for each such dimension, as shown in figure 2. In this way, such seeds provides the concept of locality because the more local the connection (i.e. $\Delta x$ close to 0.0 ), the greater the impact of the Gaussian function. This extension is called HyperNEAT with a Link Expression Output (HyperNEAT-LEO).

Each of the three variants discussed in this section provides different means of controlling connectivity and encouraging modularity. The next section introduces the experiments designed to demonstrate the differences among these approaches to thresholding. 


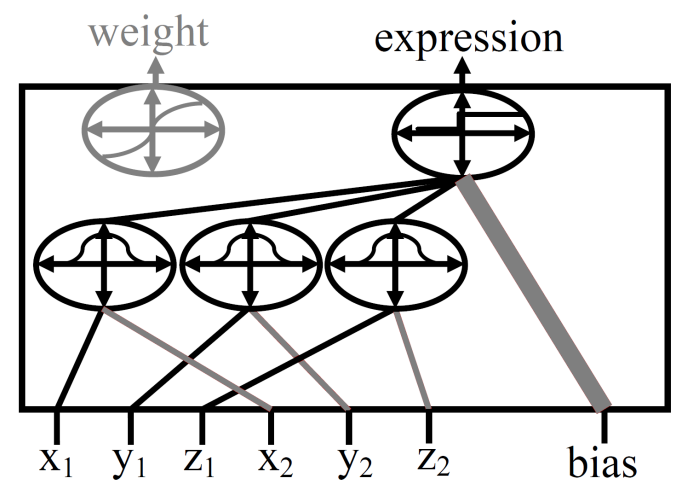

Figure 2: Seed Expressing Global Locality. Initial CPPN topologies imparted with geometric principles can seed evolution. For example, a sixdimensional CPPN can be initialized with three hidden nodes connected by positive (black) and negative (gray) weights. These hidden nodes take as input $x_{1}-x_{2}(\Delta x), y_{1}-y_{2}(\Delta y)$, and $z_{1}-z_{2}(\Delta z)$ respectively and have Gaussian activation functions. The nodes are connected to the link expression output that has a bias of -3 . Because the Gaussian function peaks at 0.0 , the expression output value is greater than or equal to 0.0 only when $\Delta x, \Delta y$, and $\Delta z$ are 0.0. Thus, the initial population is seeded with CPPNs that constrain connectivity to respect locality. When seeding in this way, the traditional weight output of the CPPN (grayed in this image) is included as usual with a set of direct connections from all the inputs (not shown).

\section{Experimental Setup}

The experiments in this paper are designed to investigate the effect of different methods of thresholding on connectivity and modularity. The key focus is 
Input: Substrate Configuration

Output: Solution CPPN

Initialize population of minimal CPPNs with random weights;

while Stopping criteria is not met do

foreach $C P P N$ in the population do

foreach Possible connection in the substrate do

Query the CPPN for weight $w$ of connection and expression value $e$;

if $e>0.0$ then

Create connection with a weight $w$;

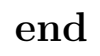

end

Run the substrate as an ANN in the task domain to ascertain fitness;

end

Reproduce CPPNs according to the NEAT method to produce the next generation;

end

Output the Champion CPPN.

Algorithm 2: HyperNEAT-LEO Algorithm

on the idea that an effective means of expressing modularity is as a function of geometry. This geometric function may be externally defined or evolved as a geometric pattern expressed by the CPPN. One key to modularity is limiting the number of connections, but limiting the connections alone does not necessarily lead to modularity. This section explains the task designed to explore how potential thresholding approaches influence modularity.

Introduced by Kashtan and Alon [11], the Retina Problem poses the chal- 
lenge of creating an ANN that must identify patterns input into the left and right retinas. The retinas consist of four inputs each that are each set to one of two values. Because the values for each input are binary, a four-input retina can take 16 possible patterns and there are overall 256 configurations for two four-input retinas. The individual retinas each have a unique set of specified patterns identified as valid or invalid that are equally divided into eight patterns each.

In the original Retina Problem introduced by Kashtan and Alon [11], the ANN is responsible for determining either (1) the left and right patterns are valid, or, (2) the left or right patterns are valid. In their version, modularity is helpful when alternating between these tasks because the functions of correctly classifying the left and right patterns are combined. An extension of these tasks, the Retina Left and Right task introduced by Clune et al. [1], removes the need to combine the decision of the modules with a logical operation; instead the ANN must separately judge as valid or invalid each retina's pattern. Standard HyperNEAT was applied to this task and was shown to struggle with producing the modularity required [1]. In fact, the most difficult of the variant tasks of Kashtan and Alon [11] for HyperNEAT in Clune et al. [1] was the Retina Left and Right task, which is thus chosen to explore modularity in this paper.

As shown in figure 3 , the substrate is configured with four layers consisting of eight input nodes, eight hidden nodes in layer one, four hidden nodes in layer two, and two output nodes. Their geometric coordinates follow from Clune et al. [1]. Note that this substrate has three dimensions $(x, y, z)$. Each of the inputs are set to either -3.0 or 3.0 , indicating off or on for each retina 
input. The left and right outputs specify the classification for the left and right retinas, respectively, where values close to -1.0 indicate invalid and values close to 1.0 indicate valid inputs. Each of the 256 possible patterns is presented to the retinas and the fitness of the solution is inversely proportional to the summed distance of the outputs from the correct values for all patterns. This set up follows Clune et al. [1].

Six treatments for controlling thresholding were evaluated on this task. The uniform threshold is evaluated with both low (0.3) and high (1.3) threshold settings. The dynamic distance threshold is evaluated with a constant of 0.45. Finally, three treatments for HyperNEAT-LEO are examined in which evolution is not seeded (but includes the LEO), evolution is seeded with a global locality pattern, and evolution is seeded with a locality pattern only along the $x$-axis. The seeded locality pattern is a CPPN with three hidden nodes with Gaussian activation functions that input $\Delta x, \Delta y$, and $\Delta z$ individually. These hidden nodes are then connected to the output that specifies whether a connection is expressed with a bias of -3.0 . The activation function of this output node is a step function that outputs 1.0 when the input is greater than 0.0 and outputs 0.0 otherwise. In this way, the initial seed specifies that only connections that are most local may be expressed (as in figure 2). Note that slight perturbations of the seed in the initial generation provide a variety of local connectivity patterns.

The alternative seed, which specifies locality along the $x$-axis, is similar to the complete locality seed, except that the hidden nodes for $\Delta y$ and $\Delta z$ are absent.

All experiments are run with an implementation of HyperNEAT in C\# 


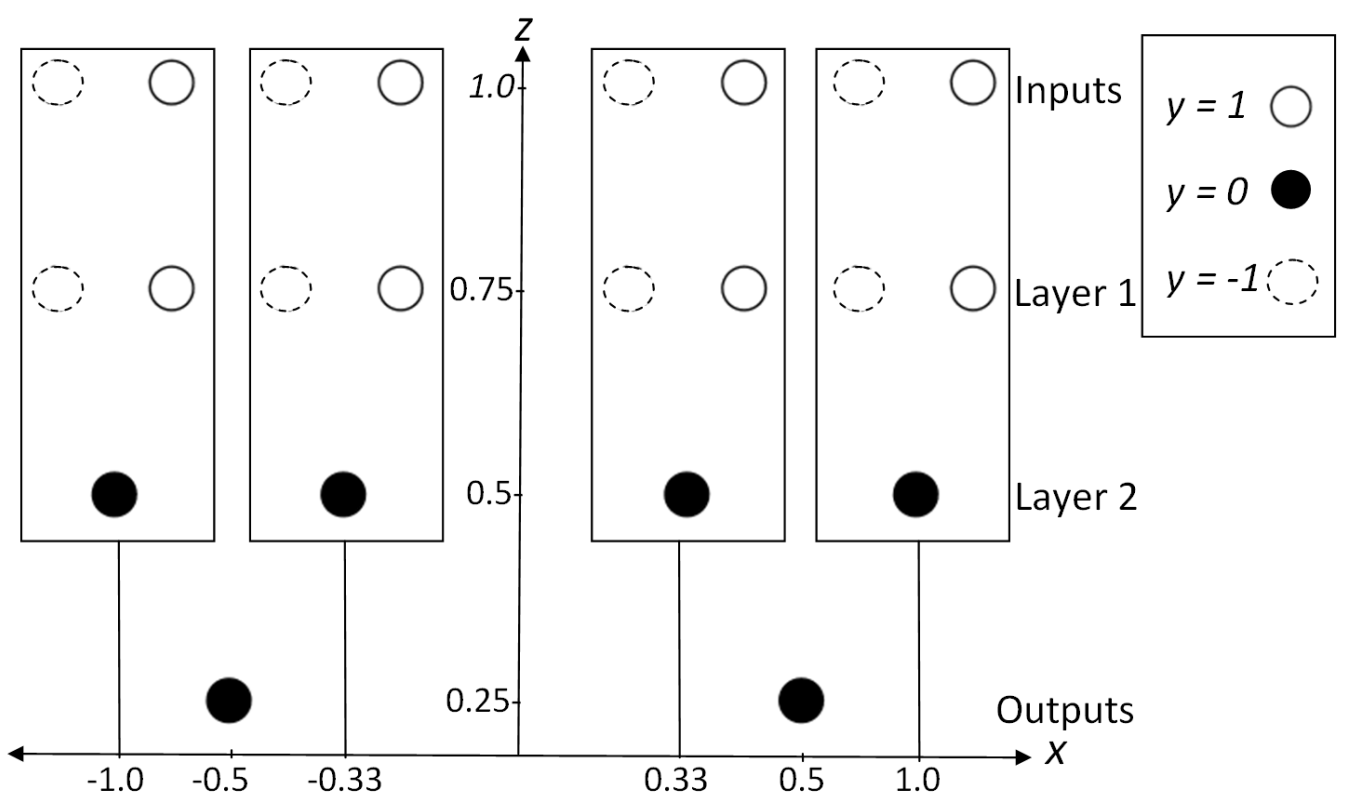

Figure 3: Substrate Configuration for the Retina Problem. The substrate configuration is set to match the setup of Clune et al. [1]. The substrate consists of four layers, with the inputs at $z=1.0$, the first hidden layer at $z=0.75$, the second hidden layer at $z=0.5$, and the outputs at $z=0.25$. Feed-forward connections are allowed between neighboring layers. Nodes in each $z$ layer are placed at an $x, y$-coordinate. The $y$ coordinates are indicated by the different circle patterns. These patterns are solid line circles for $y=1.0$, filled black circles for $y=0.0$ and dotted line circles for $y=-1.0$. The left and right sides of the retina are reflected through symmetric coordinates on the negative and positive sides the $x$-axis. This configuration provides a definite division between left and right modules at $x=0$.

by Phillip Verbancsics (available at http://eplex.cs.ucf.edu). Because HyperNEAT is an extension of NEAT, most of the parameters are the same as in 
NEAT. The population size is 500. Available CPPN activation functions are absolute value, bipolar sigmoid, Gaussian, linear, sine, and step. The probability of adding a node to the CPPN is 0.03 and the probability of adding a connection is 0.1 . The probability of asexual reproduction is 0.75 . The disjoint and excess node coefficients are both 1.0 and the weight difference coefficient is 1.0. The target number of species is 25. Finally, the elitism proportion is 0.1 .

\section{Results}

Each of the thresholding approaches are evaluated over 100 runs consisting of 5,000 generations of evolution. The performance is recorded for the champion of each generation as the percentage of the 256 patterns applied to the retinas that the ANN correctly classifies for both the left and right retina patterns (figure 4). As previously shown [1], standard HyperNEAT with any of the uniform thresholding approaches fails to achieve high average performance: The low threshold converges to $79 \%$ correct on average and the high threshold reaches $82 \%$ correct. Interestingly, the dynamic distance threshold that might be assumed to encourage modularity decreases performance, reducing the average performance to $76 \%$. HyperNEAT-LEO without a CPPN seed increases performance to $84 \%$, but does not significantly outperform the high uniform threshold. HyperNEAT-LEO with CPPN seeds representing locality (both global and only along the $x$-axis) significantly $(p<0.001$ by Student's t-test) outperform all other approaches on average, reaching $95 \%$ and $99 \%$ accuracy, respectively. 
The differing performance of each approach can also be seen in how frequently they solve the problem perfectly (figure 5). Of the 100 runs given to them, the uniform thresholds (low and high) solve the problem only $2 \%$ and $12 \%$ of the time, respectively. The dynamic distance threshold never finds a perfect solution. Evolving the LEO without a seed solves the problem as often as the high uniform threshold, $12 \%$ of the time. The main result is that seeding with locality significantly improves the ability to find the perfect modular solution. The complete locality seed solves the problem $67 \%$ of the time and the seed that specifies locality along the $x$-axis increases the rate of finding the perfect solution to $91 \%$. These results support the hypothesis that the CPPN itself should be given control of locality from a seed that begins evolution with significant locality.

A closer look at the structure of these final solutions gives an idea of how and why they succeed or fail. In the case of the uniform thresholds and the non-seeded HyperNEAT-LEO, the common outcome is to prefer fullyconnected or near fully-connected patterns (figure 6). These patterns indicate that the struggle between finding modularity and establishing the correct pattern for each retina is dominated by optimizing the weight patterns, overriding movement towards modularity. The distance threshold produces the opposite effect in its patterns (figure 7), leading to greater modularity. However, while imposing a locality bias on the weight threshold results in modular networks, the ability to find a weight pattern that is optimal is reduced (figure 5). Only when locality is provided through a LEO-CPPN seed genome does modularity become the prevailing pattern, while also allowing the weights to be optimized separately (figure 8). Hence, clearly separated 


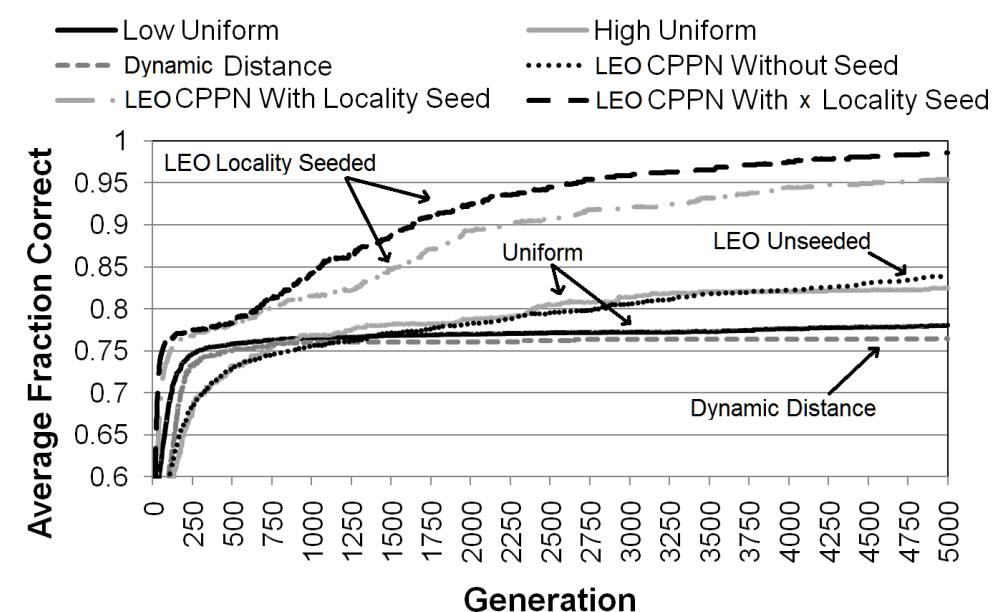

Figure 4: Average Percentage of Correct Classifications for Thresholding Methods. The fraction of correct classifications of the 256 possible patterns is averaged over 100 runs for each of the thresholding approaches. Each run lasts 5,000 generations and consists of a population of 500. The uniform and dynamic distance thresholds converge to sub-optimal values. The non-seeded LEO CPPN threshold is still improving at generation 5,000, but is not significantly outperforming the high uniform threshold. However, each of the seeded thresholds exceed the performance of the other approaches with significance $p<0.001$, demonstrating the importance of locality to the evolution of modularity.

structures emerge consistently. 


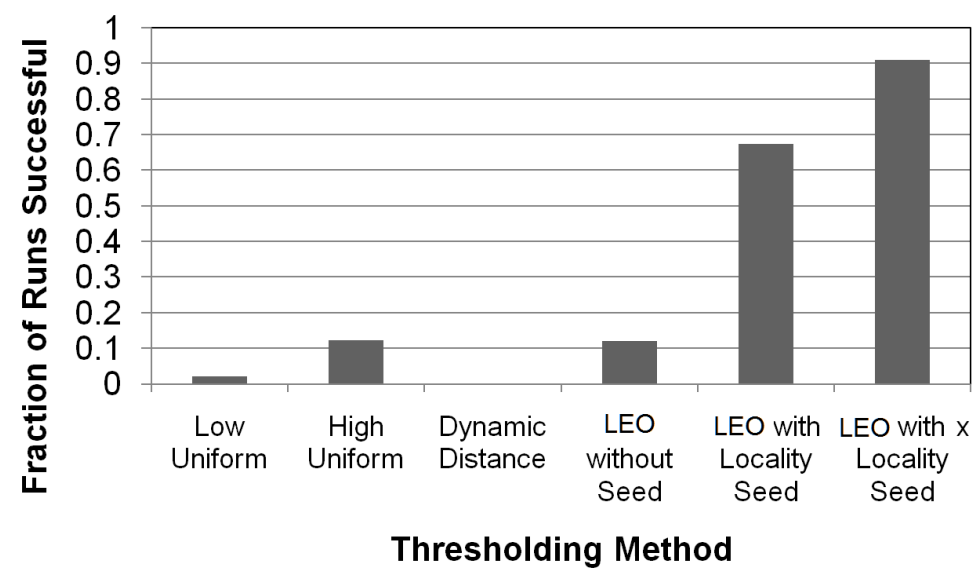

Figure 5: Fraction of Runs that are Perfect by Thresholding Method. The fraction of 100 runs that are successful in correctly classifying all of the 256 possible patterns is shown for each of the thresholding approaches after 5,000 generations. The low uniform and dynamic distance thresholds find the solution the least often, with success rates of $2 \%$ and $0 \%$, respectively. The non-seeded LEO CPPN threshold matches the high uniform threshold; both find the solution in $12 \%$ of the runs. The global locality seed improves the odds of finding the solution to $67 \%$, demonstrating how essential the concept of locality is not only to achieving a high fitness, but finding the exact solution. The $x$-axis locality seed improves upon the locality seed, finding the solution in $91 \%$ of the runs, confirming the intuition that the solution's best opportunity for modularity in this domain is along the $x$-axis.

\section{Discussion}

Modularity is a key component in generating large and complex structures, in addition to regularities and hierarchies [12]. HyperNEAT is able to gen- 


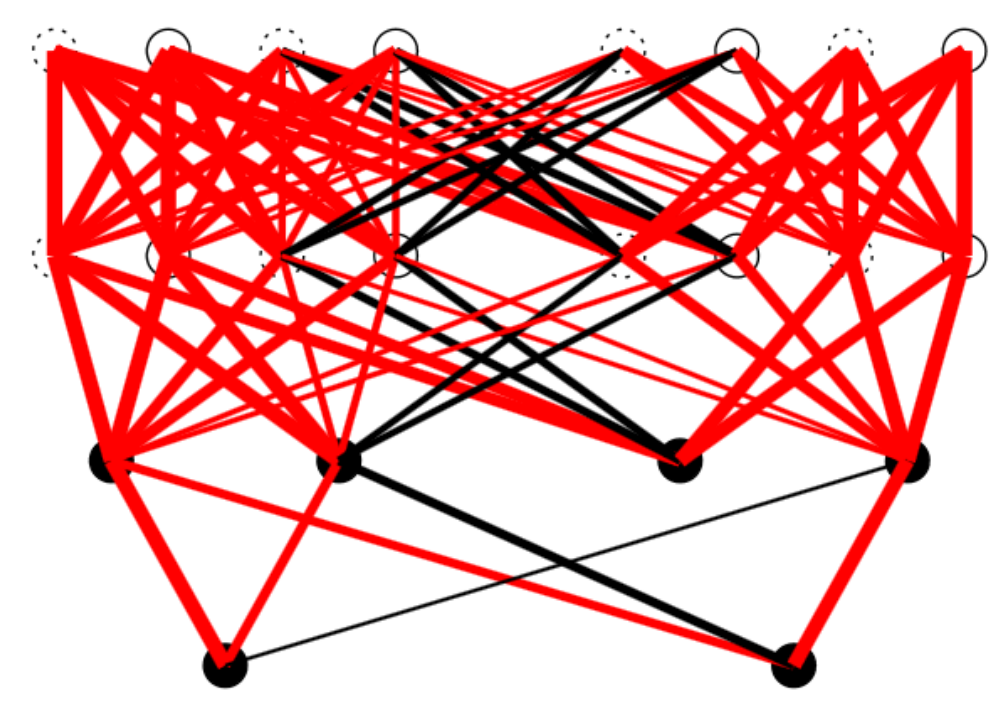

Figure 6: Typical Connectivity Pattern of Uniform Threshold. The uniform threshold fails to generate modularity. Instead, it consistently produces near fully-connected networks. These patterns are similar to those produced by the LEO without a seed. Optimizing weight patterns overrides the creation of modular structures.

erate regular patterns through a hierarchy of function compositions, but has faced a challenge producing modular patterns [1]. By modifying HyperNEAT such that the expression of connectivity is independent of the pattern of the weights, modularity and regularity can be made independent from each other. Thus both of these key features can be optimized separately. Enhancing this idea, search can be biased by seeding evolution with geometric concepts, such as locality.

One way to understand why determining whether a connection is expressed should be independent from determining its weight is to consider 


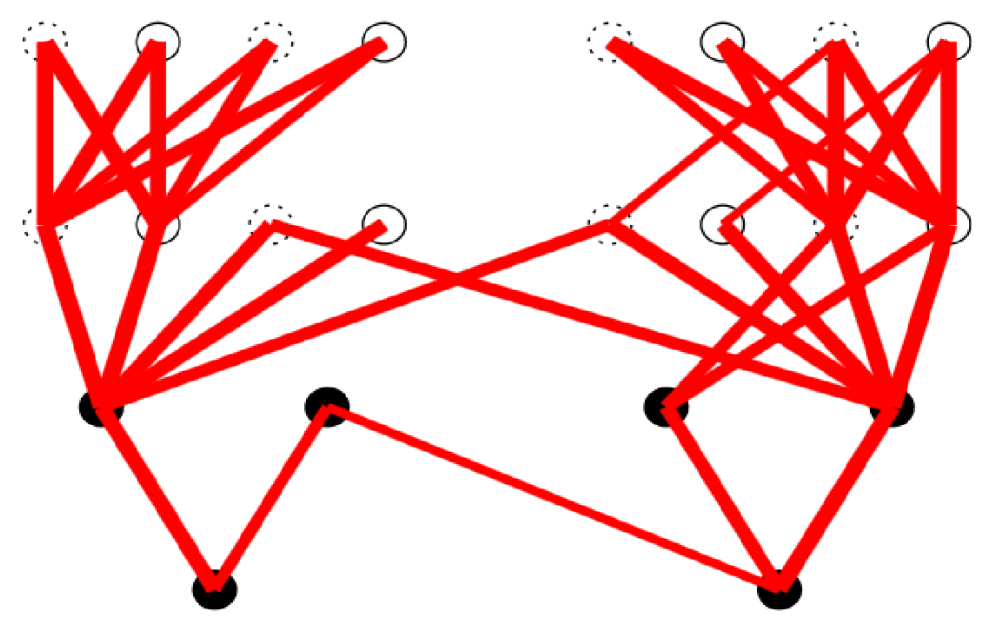

Figure 7: Typical Connectivity Pattern of Dynamic Distance Threshold. The dynamic distance threshold generates apparent modularity, but has difficulty finding the correct weight patterns. The problem is that the dynamic threshold impacts not only how connections are expressed, but the weight patterns that are created. Because longer distances mean higher thresholds, higher weight outputs become necessary, resulting in a movement towards extremes.

how connection expression and weight determination might interact when they are both determined by the same variable, as in traditional HyperNEAT. In effect, the need to suppress connections in some parts of the network can lead to distortions in the pattern of weights, which are themselves a function of the network geometry. In struggling to balance these two demands through a single function, the CPPN ultimately succeeds at neither. This insight suggests that the traditional thresholding technique in HyperNEAT was probably not the best choice in retrospect, although it has been in use 

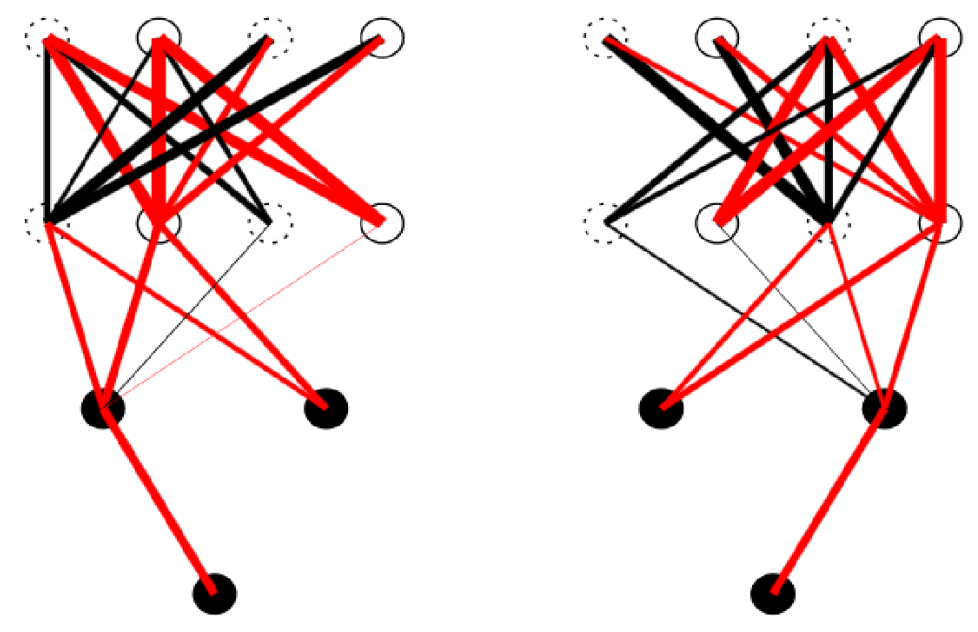

Figure 8: Typical Connectivity Pattern of LEO with Locality Seed. Modularity is commonly found when HyperNEAT-LEO is given the concept of locality. Once modularity is found, the regularities needed to solve the task for each module can be discovered in the weight pattern. This separation is possible because HyperNEAT-LEO specifies the pattern of weights and the pattern of connection expression through different outputs of the CPPN. Thus both can be evolved independently, freeing evolution from the burden of searching for a weight pattern that has both the correct weight settings to solve the problem and the necessary modularity.

for several years $[4,7]$. Nevertheless, the good news is that many interesting new structures may evolve now that this problem is uncovered.

An exciting implication of this work is that both connectivity (i.e. which connections are expressed) and connection weights can be indirectly encoded as a function of geometry to provide key prerequisites to generating complex structures. The ability to manipulate the expression of connectivity 
independently of weights provides the ability to create modules. However, modularity itself follows important geometric principles. One of these, locality, is inherent in the natural universe, that is, components that are grouped into a module are necessarily located near each other because of physical constraints. Simulated structures do not necessarily have such constraints placed upon them, but the principles that arise because of such constraints can be helpful in creating structures that resemble those of nature. Importantly, it is not enough simply to enforce the concept of locality to create modules, as demonstrated by the failure of the dynamic distance threshold. Instead, evolution should be allowed to find its own rules for modularity. This insight gives hope that HyperNEAT can now be applied to new domains that may benefit from modular neural organizations.

\section{Conclusion}

This paper investigated variations on thresholding connectivity in HyperNEAT, introducing the dynamic distance threshold and Link Expression Output. While HyperNEAT has struggled with modularity in the past, decoupling the weight pattern from whether a connection is expressed facilitated the ability to generate modular ANNs with HyperNEAT-LEO. Instead of failing to find the solution to the modular Retina Problem, HyperNEATLEO with a locality seed is almost always able to find the perfect solution, whose modularity is visually apparent in the network geometry. Thus a simple extension of HyperNEAT now captures two key features of natural systems: regularities and modularity. Ultimately, providing HyperNEAT-LEO 
with the principle of locality, but allowing evolution to alter the principle, leads to modular structures. Principles from nature such as locality combined with evolutionary algorithms may help us to someday approach the necessary ingredients to match the complexity of nature.

\section{Acknowledgements}

This research is supported in part by a Science, Mathematics, and Research for Transformation (SMART) fellowship from the American Society of Engineering Education (ASEE) and the Naval Postgraduate School.

\section{References}

[1] Jeff Clune, Benjamin E. Beckmann, Philip K. McKinley, and Charles Ofria. Investigating whether hyperneat produces modular neural networks. In Proceedings of the Genetic and Evolutionary Computation Conference (GECCO-2010), New York, NY, USA, 2010. ACM Press.

[2] Jeff Clune, Benjamin E. Beckmann, Charles Ofria, and Robert T. Pennock. Evolving coordinated quadruped gaits with the hyperneat generative encoding. In Proceedings of the IEEE Congress on Evolutionary Computation (CEC-2009) Special Section on Evolutionary Robotics, Piscataway, NJ, USA, 2009. IEEE Press. To appear.

[3] David D'Ambroiso and Kenneth O. Stanley. Evolving policy geometry for scalable multiagent learning. In Proceedings of the Ninth International Conference on Autonomous Agents and Multiagent Systems (AAMAS-2010), page 8, New York, NY, USA, 2010. ACM Press.

[4] David D'Ambrosio and Kenneth O. Stanley. A novel generative encoding for exploiting neural network sensor and output geometry. In Proceedings of the Genetic and 
Evolutionary Computation Conference (GECCO 2007), New York, NY, 2007. ACM Press.

[5] David B. D'Ambrosio and Kenneth O. Stanley. Generative encoding for multiagent learning. In Proceedings of the Genetic and Evolutionary Computation Conference (GECCO 2008), New York, NY, 2008. ACM Press.

[6] Ivan Garibay, Ozlem Garibay, and Annie Wu. Effects of module encapsulation in repetitively modular genotypes on the search space. In Proceedings of the Genetic and Evolutionary Computation Conference (GECCO 2004), pages 1125-1137, New York, NY, USA, July 2004. ACM.

[7] Jason Gauci and Kenneth O. Stanley. Generating large-scale neural networks through discovering geometric regularities. In Proceedings of the Genetic and Evolutionary Computation Conference (GECCO 2007), New York, NY, 2007. ACM Press.

[8] Jason Gauci and Kenneth O. Stanley. A case study on the critical role of geometric regularity in machine learning. In Proceedings of the Twenty-Third AAAI Conference on Artificial Intelligence (AAAI-2008), Menlo Park, CA, 2008. AAAI Press.

[9] Jason Gauci and Kenneth O. Stanley. Autonomous evolution of topographic regularities in artificial neural networks. Neural Computation, page 38, 2010.

[10] L.H. Hartwell, J.H. Hopfield, S Leibler, and A.W. Murray. From molecular to modular cell biology. Nature, 402, 1999.

[11] Nadav Kashtan and Uri Alon. Spontaneous evolution of modularity and network motifs. Proceedings of the National Academy of Sciences, 102(39):13773-13778, September 272005 .

[12] H. Lipson. Principles of modularity, regularity, and hierarchy for scalable systems. Journal of Biological Physics and Chemistry, 7, 2007.

[13] Kenneth O. Stanley. Compositional pattern producing networks: A novel abstraction of development. Genetic Programming and Evolvable Machines Special Issue on Developmental Systems, 8(2):131-162, 2007. 
[14] Kenneth O. Stanley, David B. D'Ambrosio, and Jason Gauci. A hypercube-based indirect encoding for evolving large-scale neural networks. Artificial Life, 15, 2009. To appear.

[15] Kenneth O. Stanley and Risto Miikkulainen. Evolving neural networks through augmenting topologies. Evolutionary Computation, 10:99-127, 2002.

[16] Kenneth O. Stanley and Risto Miikkulainen. A taxonomy for artificial embryogeny. Artificial Life, 9(2):93-130, 2003.

[17] Kenneth O. Stanley and Risto Miikkulainen. Competitive coevolution through evolutionary complexification. Journal of Artificial Intelligence Research, 21:63-100, 2004.

[18] Matthew E. Taylor, Shimon Whiteson, and Peter Stone. Comparing evolutionary and temporal difference methods in a reinforcement learning domain. In Proceedings of the Genetic and Evolutionary Computation Conference (GECCO 2006), pages 13211328, New York, NY, July 2006. ACM Press.

[19] A. M. Turing. The Chemical Basis of Morphogenesis. Royal Society of London Philosophical Transactions Series B, 237:37-72, August 1952.

[20] Phillip Verbancsics and Kenneth O. Stanley. Evolving static representations for task transfer. Journal of Machine Learning Research, 11, 2010.

[21] G.P. Wagner and L. Altenberg. Complex adapatations and the evolution of evolovability. Evolution, 50, 1996.

[22] Shimon Whiteson. Improving reinforcement learning function approximators via neuroevolution. In $A A M A S$ '05: Proceedings of the fourth international joint conference on Autonomous agents and multiagent systems, pages 1386-1386, New York, NY, USA, 2005. ACM.

[23] Shimon Whiteson and Daniel Whiteson. Stochastic optimization for collision selection in high energy physics. In IAAI 200\%: Proceedings of the Nineteenth Annual Innovative Applications of Artificial Intelligence Conference, Vancouver, British Columbia, Canada, July 2007. AAAI Press. 
[24] R. Paul Wiegand, Gautham Anil, Ivan Garibay, Ozlem Garibay, and Annie Wu. On the performance effects of unbiased module encapsulation. In Proceedings of the Genetic and Evolutionary Computation Conference (GECCO 2009), pages 17291736, New York, NY, USA, July 2009. ACM. 